\title{
Solute delivery as function of catchment conditions across timescales
}

\author{
JULIA L.A. KNAPP ${ }^{1,2}$, JANA VON FREYBERG ${ }^{2,3,4}$ AND \\ JAMES KIRCHNER ${ }^{2,4}$ \\ ${ }^{1}$ Durham University \\ ${ }^{2}$ ETH Zurich \\ ${ }^{3} \mathrm{EPFL}$ \\ ${ }^{4}$ Swiss Federal Institute for Forest, Snow and Landscape \\ Research WSL \\ Presenting Author: julia.1.knapp@durham.ac.uk
}

Solute concentrations in streamwater are known to vary with discharge depending on the solutes' predominant sources in the catchment. Studying the cQ behavior, i.e. the response of streamwater chemistry to changes in discharge, can thus improve our understanding of how catchments store and release water and solutes.

Here, we investigate variations in $\mathrm{cQ}$ relationships on longand intra-event timescales, using high-frequency measurements spanning a wide range of solutes in a pre-alpine Swiss catchment. We determined both long-term $\mathrm{cQ}$ relationships (from the entire 2-year high-frequency data set) and intra-event $\mathrm{cQ}$ relationships (from high-frequency measurements of 30 individual events). This analysis provides insights into how intraevent and longer-term cQ relationships may differ, providing different perspectives on processes regulating transport through the landscape. Unsurprisingly, groundwater-sourced solutes exhibited dilution patterns at all time scales, and thus their longterm cQ behavior was also representative of their cQ behavior during hydrologic events. Other solutes, however, exhibited highly variable cQ behavior from one event to the next, and very different $c Q$ patterns at event and longer-term time scales.

Most of the observed event-to-event variability in cQ behavior could be linked to factors such as catchment wetness, event size, and event-water contributions, highlighting their importance for the mobilization of solutes. We thus hypothesized that antecedent conditions, rather than discharge per se, may shape solute mobilization and delivery (and thus cQ relationships) on longer timescales as well. To test this hypothesis, we compared cQ relationships as functions of antecedent wetness and precipitation intensity, using long-term, low-frequency data from small to mid-sized catchments in Switzerland and the UK. Similar to the analysis of the high-frequency data, we observed substantial differences in $\mathrm{cQ}$ relationships as function of catchment conditions across solutes and sites. This highlights the importance of catchment conditions for flowpath partitioning and solute mobilization from different pools on different timescales. Our analysis thus provides a framework that allows for inferring dominant catchment hydrological processes (catchment sources, pathways, and travel times) from cQ behavior. 